\title{
Reliability and reproducibility of Atlas information
}

\author{
Thomas Breuer, Gunter Malle, and E.A. O’Brien \\ ABstract. We discuss the reliability and reproducibility of much of the in- \\ formation contained in the $\mathbb{A} \mathbb{T L} \mathbb{A}$ of Finite Groups.
}

\section{Introduction}

The ATLAS of Finite Groups [7, published in 1985, contains a wealth of information on the sporadic simple groups, their covering groups and automorphism groups, as well as on numerous other finite simple groups of small order. It has become an indispensable tool for researchers not only in finite group theory but in many other areas where finite groups play a role. In a recent letter, Jean-Pierre Serre stated that he "can't think of any other book published in the last 50 years which had such an impact", while Benedict Gross is cited as saying that if ever the university library caught fire and one could save just one book, the obvious choice would be the $\mathbb{A} \mathbb{T L A S}$. In view of this, the question of reliability and reproducibility of the results stated there is of considerable importance, particularly since the $\mathbb{A} \mathbb{T L} \mathbb{S}$ does not contain proofs of the information it records, although for the sporadic groups it gives a list of references from which some of the stated results are taken.

In the intervening thirty years, some misprints and errors have been found in the $\mathbb{A} T L A S$. Corrections and improvements known at the time of publication of the Atlas of Brauer Characters $1 \mathbf{1 1}$ are listed in an appendix [6] to that book; the website [15] reports those found later. No corrections have been added since 2002.

Few of these concern the actual character tables; apart from the misprints, only three cases are known in which the printed tables contain mathematical mistakes not arising simply from inconsistencies concerning irrationalities and power maps; these concern the nearly simple groups $2 . \mathrm{L}_{4}(3) .2_{3}, \mathrm{U}_{3}(11) .2$ and $2 . \mathrm{U}_{6}(2) .2$; see the more detailed comments in Section 3.1

Our purpose is to provide references for some of the cited results; to report on the independent reconstruction of most of the character table information (see Theorem 3.1) and on the methods used to do so; and to describe how such a check could be carried out independently by anyone having available sufficient computing power.

2000 Mathematics Subject Classification. Primary 20C15, 20-00, 20-04; Secondary 20D05, 20F30.

(c) 0000 (copyright holder) 
The $\mathbb{A} \mathbb{T} \mathbb{A} \mathbb{S}$ contains two essential pieces of information about each simple group $S$ it lists: the ordinary character tables of all (or most) bicyclic extensions M.S.A of $S$, and all (or most) of the maximal subgroups of all subgroups $S . A$ of the automorphism group of $S$ that contain $S$. We discuss only these two pieces of data, and do not consider the other information also given in the $\mathbb{A} \mathbb{L} \mathbb{A} \mathbb{S}$, such as constructions of the groups and their presentations, since we consider these two as the information most widely used. As we will explain below, the situation for published proofs and references for maximal subgroups is very satisfactory, so we mostly deal with the question of (re)constructing the ordinary character tables. Here, we will not try to follow the original proofs but rather give a modern approach, which builds on the use of computer programs and is highly automatic (and so, we claim, is much more reliable than hand calculations).

One further comment is in order: nowadays, all the character tables contained in the $\mathbb{A} \mathbb{T} \mathbb{L} \mathbb{S}$, incorporating the corrections, and many more, are stored electronically in the character table library [3] of the computer algebra system GAP [23]. Our checking will be with respect to these electronic tables.

The ATLAS tables in GAP's character table library have been constructed from the data files (in the so-called "Cambridge format") which were used also to create the printed $\mathbb{A} \mathbb{T L A S}$; hence the ordering of rows and columns in the GAP tables coincides with the $\mathbb{A} \mathbb{T} \mathbb{L} \mathbb{A}$ S ordering. We do not know how far the old "Cambridge format" files represent exactly the contents of the printed $\mathbb{A} \mathbb{T} \mathbb{L} \mathbb{S}$. It might be possible to scan the printed $\mathbb{A} \mathbb{T} \mathbb{A} \mathbb{S}$ and to turn the result into GAP files; these could then be compared with the GAP tables; but we did not consider worthwhile following this cumbersome procedure here. So for this practical reason all statements on accuracy and consistency will only be relative to the electronic GAP versions of the $\mathbb{A} \mathbb{T} \mathbb{A} S$, and we make no claim on their agreement with the original printed version.

The same data format has been used for the Brauer character tables in [11]: the information in $\mathbf{1 1}$ depends on the $\mathbb{A} \mathbb{T} \mathbb{L} \mathbb{A}$, the Brauer character tables can be understood only relative to the ordinary $\mathbb{A} \mathbb{T L} \mathbb{A} S$ character tables. When the Brauer character tables in [11] were prepared for both the printed version and their inclusion in GAP's character table library, the ordinary $\mathbb{A} \mathbb{T} \mathbb{A} \mathbb{S}$ tables were already checked systematically, and many of the errors listed in [6] were found by these checks.

Acknowledgement: We thank Jean-Pierre Serre for raising the question of reliability of $\mathbb{A} \mathbb{T} \mathbb{A} \mathbb{S}$ information, which led to the current paper, and for comments on a preliminary version.

\section{Maximal subgroups}

We begin by discussing the information in the $\mathbb{A} \mathbb{L} \mathbb{A} S$ concerning maximal subgroups of simple groups and of their automorphism groups. The situation here is rather favourable, in the sense that published proofs for all of the lists of maximal subgroups as printed in the $\mathbb{A} \mathbb{L} \mathbb{A} \mathbb{S}$ (modulo the corrections listed in [15]) are available. For the sporadic simple groups and their automorphism groups, references for the stated results are already given in the original version of the $\mathbb{A} \mathbb{T} \mathbb{A} \mathbb{S}$, and new information obtained since then is referenced in [15]. See [26 for a survey of this topic. 
As for the alternating groups $\mathfrak{A}_{n}, n \leq 13$, the question clearly is about their primitive (maximal) subgroups, and these are well-known, see e.g. 14 for a classical reference, or 21. The simple groups of Lie type in the $\mathbb{A T L A S}$ are of small Lie rank, and most are of classical type. For the latter, much information on maximal subgroups had already been accumulated in pre-ATLAS time, starting with the work of Galois; the recent book by Bray, Holt and Roney-Dougal 2 gives complete proofs of the lists of maximal subgroups, and does not rely on the ATLAS lists. For the series of exceptional groups of types ${ }^{2} B_{2},{ }^{2} G_{2}, G_{2}$ and ${ }^{3} D_{4}$, there exist published proofs [12, 13, 22 ; for ${ }^{2} F_{4}(2)^{\prime}$ and $F_{4}(2)$ proofs can be found in [16, 25. Finally, for ${ }^{2} E_{6}(2)$ the $\mathbb{A} \mathbb{T L} \mathbb{S}$ does not claim to give complete information.

Thus, complete independent proofs for the maximal subgroup information in the $\mathbb{A} \mathbb{T L} \mathbb{S}$ are now available in the literature.

\section{Character tables}

We now turn to the more problematic question of character tables and their correctness.

The tables for alternating groups, symmetric groups and their covering groups are known by published classical work of Frobenius and Schur (see [19] and the references therein).

For many of the sporadic groups the $\mathbb{A} T L \mathbb{A} S$ references published proofs, for example a paper of Frobenius [8] for the Mathieu groups. (As Serre pointed out to us, while Frobenius sketched how the character tables were constructed, he said nothing about conjugacy classes. This is interesting, because even the existence of $M_{24}$ was not completely clear at that time.) However, such references do not exist for all of the sporadic groups.

For the groups of Lie type, the situation is even more opaque. No references are given in the $\mathbb{A} T L \mathbb{A}$ S. While there are published tables for some series of groups (for example for $\mathrm{SL}_{2}(q)$ by Schur [18, for ${ }^{2} B_{2}\left(q^{2}\right)$ by Suzuki [22], and for $\mathrm{SL}_{3}(q)$ and $\mathrm{SU}_{3}(q)$ by Simpson and Frame [20, to mention just a few), most of the tables for the groups of larger rank and in particular for their decorations were computed using ad hoc techniques by the $\mathbb{A} \mathbb{T L} \mathbb{S}$ authors. Even today, the representation theory of finite groups of Lie type, despite the tremendous achievements of George Lusztig, is not capable of predicting the complete character tables of all the groups listed in the $\mathbb{A} \mathbb{L L} \mathbb{S}$, in fact not even the character degrees in all cases.

3.1. Known mistakes. Let us start by discussing the nature of known mistakes: there are 142 entries marked as errors (three stars) in [6], and 17 such entries in 15. Many of the errors affect the descriptions of group constructions or maximal subgroups, or indicator signs or power maps, and 27 concern character values. Some of them could be fixed also by just changing power maps and some can be detected by testing orthogonality relations - for example, five columns of the character table of $6 . F i_{22} .2$ were not printed, and a character value -1 of $G_{2}(4)$ was listed as 1 . Some errors concern inconsistencies among several characters. Consider for example the error on page 128 (the group in question is the sporadic group $S u z)$ : the improvements list states "Change sign of $i 3$ in $\chi_{7}, \chi_{8}, \chi_{18}, \chi_{19}, \chi_{21}, \chi_{22}$ on $6 B, 6 C$ ". For the simple group, one could keep the character values, and adjust the power maps instead. However, then one would have to change character values in central extensions of $S u z$. For $G=3 \cdot \mathrm{U}_{3}(8) \cdot 3_{1}$ and $G=3 \cdot \mathrm{U}_{3}(8) \cdot 3_{2}$, the problem was the consistent choice of irrationalities in the faithful characters on the outer 
classes - extensions to $G$ of some faithful irreducible characters of the derived subgroup were multiplied by 9 -th roots of unity, thus each of the shown characters exists for a suitable group of the given structure but they do not fit to the same isomorphism type. But there are tables of (non-simple) groups which are wrong in a more serious way, in the sense that characters were listed that cannot exist. A wrong splitting of classes is shown for 2. $\mathrm{L}_{4}(3) .2_{3}$; for both $G=\mathrm{U}_{3}(11) .2$ and $G=2 . \mathrm{U}_{6}(2) .2$, the extensions to $G$ of two irreducible characters of different degree of the derived subgroup were interchanged. These mistakes are consistent with the orthogonality relations and so are much harder to spot.

3.2. Recomputing tables automatically. We now propose our approach to reconstructing most of the character tables in the (electronic version of the) $\mathbb{A} \mathbb{T} \mathbb{A} \mathbb{S}$ in a reproducible and essentially automatic way. It relies on a powerful algorithm by Unger [24. We use his implementation which is available in MAGma 1]; it uses no precomputed tables and does not rely on $\mathbb{A} \mathbb{T} \mathbb{L} \mathbb{S}$ bounds or data. In the current version of GAP $\mathbf{2 3}$, the standard method to compute a character table is the less powerful Dixon-Schneider algorithm [17. Both take as input a faithful representation of a finite group, either as a permutation group or as a matrix group over some finite field, and automatically compute the ordinary character table of that group, including in particular the list of conjugacy classes, their sizes and the power map on the classes.

Now assume that we want to reconstruct the character table information for a finite simple group $S$ appearing in the $\mathbb{A} \mathbb{T} \mathbb{A} \mathbb{S}$. We proceed as follows. First, the size and structure of the automorphism group $\operatorname{Aut}(S)$, the Schur multiplier $M(S)$, and the action of the first on the second are well-known; see, for example, 9 . From this it is possible to compile a list of all bicyclic extensions $G=M . S . A$ for $S$ as considered in the $\mathbb{A} \mathbb{T} \mathbb{A} \mathbb{S}$ : namely, both $M \leq M(S)$ and $A \leq \operatorname{Out}(S)$ are cyclic and $G$ is an extension by $A$ of the central extension M.S of $S$.

3.3. Enumerating bicyclic extension. Let $G$ be a group that contains normal subgroups $M<N$, and consider the set of subquotients of the form $U / K$ with the property that $N \leq U, K \leq M, K$ is normal in $U$, and both $U / N$ and $M / K$ are cyclic. The group $G / N$ acts on this set by conjugation, and a set of class representatives under this action contains all bicyclic extensions of $N / M$ that occur as subquotients of $G$, up to isomorphism. (Some representatives may in fact be isomorphic; if we are interested in representatives up to isomorphism, we must check this case by case.)

If $N$ is a Schur cover of a finite simple group $S$, so $M$ is the Schur multiplier of $S$, and $G / M$ is isomorphic to the automorphism group of $S$, then a set of class representatives yields all bicyclic extensions of $S$, up to isoclinism. (Again, the set may be too large.) We discuss the three most complicated cases occurring in the $\mathbb{A} \mathbb{T L} \mathbb{A}$ in more detail.

3.3.1. Case 1: $S=\mathrm{L}_{3}(4)$. The Schur multiplier $M$ and the outer automorphism group $A$ of $S$ have the structures $3 \times 4^{2}$ and $D_{12}$ (the dihedral group of order twelve), respectively. A group $G$ of the structure M.S.A as mentioned above exists. Since the subgroups $M_{1}$ and $M_{2}$ of order three and 16 in $M$ are characteristic in $G$, we may consider the bicyclic extensions of $S$ that occur as subquotients of $G / M_{1}$ and $G / M_{2}$, and then get the general bicyclic extensions of $S$ that occur as subquotients of $M$ as subdirect products. 
First we fix the notation for the cyclic subgroups of $G / N$. The unique cyclic subgroup of order six is called 6 by the $\mathbb{A T L A S}$, its subgroups of order two (the centre of the dihedral group) and three are called $2_{1}$ and 3, respectively, and representatives of the other conjugacy classes of subgroups of order two are called $2_{2}$ and $2_{3}$.

- The group $G / M_{1}$ has the structure $4^{2} . S . D_{12}$. Let $M / M_{1}$ be generated by commuting elements $a, b$ of order four, and let $c=(a b)^{-1}$. As stated in [7, p. 23], the outer automorphism group $G / N$ of $S$ acts as follows on $M / M_{1}$ :

$$
\begin{array}{llll}
6: & a \mapsto b^{3}, & b \mapsto c^{3}, & c \mapsto a^{3} \\
2_{2}: & a \mapsto a, & b \mapsto c, & c \mapsto b \\
2_{3}: & a \mapsto a^{3}, & b \mapsto c^{3}, & c \mapsto b^{3}
\end{array}
$$

The three subgroups of index two in $M / M_{1}$ are $\left\langle a, b^{2}\right\rangle,\left\langle b, c^{2}\right\rangle$, and $\left\langle c, a^{2}\right\rangle$. Their normalisers in $G / N$ are the three Sylow 2 -subgroups. One of them contains the involutions $2_{1}, 2_{2}, 2_{3}$, thus we get the bicyclic extensions 2.S. $2_{1}, 2 . S .2_{2}$, and $2 . S .2_{3}$.

(The other two Sylow 2-subgroups of $G / N$ contain $2_{1}$ and conjugates of $2_{2}$ and $2_{3}$. Thus we get conjugate bicyclic extensions $2^{\prime} . S .2_{1}, 2^{\prime} . S .2_{2}^{\prime}$, $2^{\prime} . S .2_{3}^{\prime}, 2^{\prime \prime} . S .2_{1}, 2^{\prime \prime} . S .2_{2}^{\prime \prime}$, and $\left.2^{\prime \prime} . S .2_{3}^{\prime \prime}.\right)$

The group $G / N$ has the two orbits

$$
\{\langle a\rangle,\langle b\rangle,\langle c\rangle\},\left\{\left\langle a b^{2}\right\rangle,\left\langle b c^{2}\right\rangle,\left\langle c a^{2}\right\rangle\right\}
$$

on the six cyclic subgroups of order four in $M / M_{1}$. We get two nonisomorphic central extensions of $S$ by a cyclic group of order four. Both extensions are normalised but not centralised by $2_{1}$, which inverts all elements in $M / M_{1}$.

The second one, $\left(N / M_{1}\right) /\left\langle a b^{2}\right\rangle$, is called $4_{1} . S$ by the $\mathbb{A} \mathbb{T L A S}$; it is centralised by $2_{3}$, its normaliser in $G / N$ is the elementary abelian group generated by $2_{1}$ and $2_{3}$. This yields the extensions $4_{1} . S .2_{1}, 4_{1} . S .2_{2}$, and $4_{1} . S .2_{3}$.

The first one, $\left(N / M_{1}\right) /\langle a\rangle$, is called $4_{2} . S$ by the $\mathbb{A T L A S}$; it is centralised by $2_{2}$, its normaliser is the elementary abelian group generated by $2_{1}$ and $2_{2}$. This yields the extensions $4_{2} . S .2_{1}, 4_{2} . S .2_{2}$, and $4_{2} . S .2_{3}$.

(In both cases, the other two orbit points are stabilised by the other two Sylow 2-subgroups of $G / M_{1}$, which yields the conjugate bicyclic extensions $4_{1}^{\prime} . S .2_{1}, 4_{1}^{\prime} . S .2_{2}^{\prime}, 4_{1}^{\prime} . S .2_{3}^{\prime}, 4_{1}^{\prime \prime} . S .2_{1}, 4_{1}^{\prime \prime} . S .2_{2}^{\prime \prime}, 4_{1}^{\prime \prime} . S .2_{3}^{\prime \prime}, 4_{2}^{\prime} . S .2_{1}, 4_{2}^{\prime} . S .2_{2}^{\prime}$, $4_{2}^{\prime} \cdot S .2_{3}^{\prime}, 4_{2}^{\prime \prime} \cdot S .2_{1}, 4_{2}^{\prime \prime} \cdot S .2_{2}^{\prime \prime}$, and $4_{2}^{\prime \prime} \cdot S .2_{3}^{\prime \prime}$.)

- The group $G / M_{2}$ has the structure 3.S.D $D_{12}$; the centraliser of $M / M_{2}$ in $G / N$ is the cyclic subgroup of order six, conjugation with the other elements of $G / N$ inverts $M / M_{2}$.

Thus we get the bicyclic extensions 3.S, 3.S.2 $2_{1}, 3 . S .3,3 . S .6,3 . S .2_{2}$, and 3.S. $2_{3}$, and their factor groups $S, S .2_{1}, S .3, S .6, S .2_{2}, S .2_{3}$, which are pairwise nonisomorphic.

- Putting the pieces together, we get also bicyclic extensions in which the cyclic normal subgroup has order 6 or 12. Each of the above extensions with normal cyclic subgroup of order two or four and commutator factor group acting like one of the seven involutions can be combined with an 
extension with normal cyclic subgroup of order three and the same action of the commutator factor group.

In summary, we get the following pairwise nonisomorphic bicyclic extensions: $6 . S$, 6.S.2 $2_{1}, 6 . S .2_{2}, 6 . S .2_{3}, 12_{1} . S, 12_{1} . S .2_{1}, 12_{1} . S .2_{2}, 12_{1} . S .2_{3}$, $12_{2} . S, 12_{2} . S .2_{1}, 12_{2} . S .2_{2}, 12_{2} . S .2_{3}$.

3.3.2. Case 2: $S=\mathrm{U}_{4}(3)$. The Schur multiplier $M$ and the outer automorphism group $A$ of $S$ have the structures $3^{2} \times 4$ and $D_{8}$ (the dihedral group of order eight), respectively. A group $G$ of the structure M.S.A as mentioned above exists. Since the subgroups $M_{1}$ and $M_{2}$ of order four and nine in $M$ are characteristic in $G$, we may consider the bicyclic extensions of $S$ that occur as subquotients of $G / M_{1}$ and $G / M_{2}$, and then get the general bicyclic extensions of $S$ that occur as subquotients of $M$ as subdirect products.

First we fix the notation for the cyclic subgroups of $G / N$. The unique cyclic subgroup of order four is called 4 by the $\mathbb{A T L} \mathbb{A}$, its subgroup of order two (the centre of the dihedral group) is called $2_{1}$, and representatives of the other conjugacy classes of involutions are called $2_{2}$ and $2_{3}$.

- The group $G / M_{1}$ has the structure $3^{2} . S . D_{8}$. We identify $M / M_{1}$ with a 2 -dimensional vector space over $\mathbb{F}_{3}$. The action of $G / N$ on this vector space is given by the matrices

$$
\left[\begin{array}{rr}
-1 & 0 \\
0 & 1
\end{array}\right],\left[\begin{array}{ll}
0 & 1 \\
1 & 0
\end{array}\right]
$$

for the involutions from $2_{2}$ and $2_{3}$, respectively. The action of $2_{1}$ is given by the square of their product, which is the negative of the identity matrix; thus $2_{1}$ inverts all elements in $M / M_{1}$. The group $G / N$ has the two orbits

$$
\{ \pm[1,0], \pm[0,1]\},\{ \pm[1,1], \pm[1,-1]\}
$$

on the nonidentity elements of $M / M_{1}$. We get two nonisomorphic central extensions of $S$ by a cyclic group of order three.

The first one, $\left(N / M_{1}\right) /\langle[1,0]\rangle$, is called $3_{1} . S$ by the $\mathbb{A} \mathbb{T L} \mathbb{A}$ S; it is centralised by $2_{2}$ and normalised by the elementary abelian group generated by $2_{1}$ and $2_{2}$. The third subgroup of order two in this subgroup is called $2_{2}^{\prime}$, it centralises the conjugate extension $\left(N / M_{1}\right) /\langle[0,1]\rangle$, which is called $3_{1}^{\prime} . S$.

The second one, $\left(N / M_{1}\right) /\langle[1,1]\rangle$, is called $3_{2} . S$ by the $\mathbb{A} \mathbb{T L} \mathbb{A}$; it is centralised by $2_{3}$ and normalised by the elementary abelian group generated by $2_{1}$ and $2_{3}$. The third subgroup of order two in this subgroup is called $2_{3}^{\prime}$, it centralises the conjugate extension $\left(N / M_{1}\right) /\langle[1,-1]\rangle$, which is called $3_{2}^{\prime} . S$.

Thus we get the following pairwise nonisomorphic bicyclic extensions: $3_{1} . S, 3_{1} . S .2_{1}, 3_{1} . S .2_{2}, 3_{1} . S .2_{2}^{\prime}, 3_{2} . S, 3_{2} . S .2_{1}, 3_{2} . S .2_{3}$ and $3_{2} . S .2_{3}^{\prime}$.

(Note that the centre of the groups $3_{1} . S .2_{2}$ and $3_{2} . S .2_{3}$ has order three, the other four groups have trivial centre. Analogously, the conjugate bicyclic extensions $3_{1}^{\prime} \cdot S .2_{2}^{\prime}$ and $3_{2}^{\prime} \cdot S \cdot 2_{3}^{\prime}$ have centres of order three, and the centres of $3_{1}^{\prime} . S .2_{1}, 3_{1}^{\prime} . S .2_{2}, 3_{2}^{\prime} . S .2_{1}$, and $3_{2}^{\prime} . S .2_{3}$ are trivial.)

- The group $G / M_{2}$ has the structure 4.S.D $D_{8}$; the centraliser of $M / M_{2}$ in $G / N$ is the cyclic subgroup of order four, conjugation with the other elements of $G / N$ inverts $M / M_{2}$. 
Thus we get the bicyclic extensions 4.S, 4.S.2. $2_{1}$, 4.S.4, 4.S.2. $2_{2}$, and 4.S. $2_{3}$, and their factor groups $2 . S, 2 . S .2_{1}, 2 . S .4,2 . S .2_{2}, 2 . S .2_{3}, S, S .2_{1}$, $S .4, S .2_{2}, S .2_{3}$, which are pairwise nonisomorphic.

- Putting the pieces together, we get also bicyclic extensions in which the cyclic normal subgroup has order 6 or 12. Each of the above extensions with normal cyclic subgroup of order three and commutator factor group acting like one of the five involutions can be combined with an extension with normal cyclic subgroup of order two or four and the same action of the commutator factor group.

In summary, we get the following pairwise nonisomorphic bicyclic extensions: $6_{1} . S, 6_{1} . S .2_{1}, 6_{1} . S .2_{2}, 6_{1} . S .2_{2}^{\prime}, 6_{2} . S, 6_{2} . S .2_{1}, 6_{2} . S .2_{3}, 6_{2} . S .2_{3}^{\prime}$, $12_{1} . S, 12_{1} . S .2_{1}, 12_{1} . S .2_{2}, 12_{1} . S .2_{2}^{\prime}, 12_{2} . S, 12_{2} . S .2_{1}, 12_{2} . S .2_{3}, 12_{2} . S .2_{3}^{\prime}$.

The tables of $12_{1} . S .2_{2}^{\prime}$ and $12_{2} . S .2_{3}^{\prime}$ will be available in the next public release of 3 .

3.3.3. Case 3: $S=\mathrm{U}_{3}(8)$.

- The outer automorphism group $A$ of $S$ has the structure $3 \times S_{3}$. The $\mathbb{A} T L A \mathbb{S}$ notation for the four subgroups of order three in $A$ is $3_{1}$ for the central one, $3_{2}$ for the noncentral normal one, and $3_{3}$ and $3_{3}^{\prime}$ for the remaining two, which are conjugate in $A$. In addition, $A$ contains nontrivial cyclic subgroups of order two and six, in each case three conjugates called $2,2^{\prime}, 2^{\prime \prime}$ and $6,6^{\prime}, 6^{\prime \prime}$, respectively. This yields the automorphic extensions $S, S .2, S .3_{1}, S .3_{2}, S .3_{3}$, and $S .6$.

- The Schur multiplier $M$ of $S$ has order three, and a group of the structure M.S.S $S_{3}$ exists. (There is no group of the structure M.S.A.) This yields the bicyclic extensions 3.S, 3.S.3 1 , and 3.S.2; the latter is conjugate and thus isomorphic to $3 . S .2^{\prime}$ and $3 . S .2^{\prime \prime}$. Also a group of the structure $3 . S .3_{2}$ exists. Concerning groups of the structure 3.S.6, there is one such group that contains a given 3.S.3 1 type group as a subgroup of index two. As stated in [6, the groups 3.S.6' and 3.S.6" $6^{\prime \prime}$, whose existence is claimed in [7, p. 66], are the extensions of the isoclinic variants of $3 . S .3_{1}$ by their unique outer automorphisms of order two. Thus 3.S.6 and 3.S.6" are not isomorphic and in particular not conjugate to $3 . S .6$ in some larger group. We can, however, ignore them since we are interested in the bicyclic extensions only up to isoclinism.

3.4. Our algorithm. For each bicyclic extension $G$ of a simple $\mathbb{A} \mathbb{T} L \mathbb{A} S$ group $S$, we proceed as follows:

(1) We construct, or find in an existing database, a faithful representation $H$ of $G$. For groups of Lie type, it is often easy to construct such a representation from its very definition; both GAP and MAGMA provide access to natural representations. At this point, we do not actually need to establish that $H \cong G$. This will only occur at a later stage.

(2) We then give this representation to the character table algorithm in the computer algebra system of our choice (in our case MAGMA).

(3) From the output (the character table of $H$ ) we can read off the composition factors of $H$, assuming the classification of finite simple groups, its derived subgroup and the centre of that subgroup. The very few cases of simple groups of identical order can easily be distinguished by additional 
information on centraliser orders, for example. In many cases, this will already prove that $H$ is isomorphic to $G$.

(4) It is then an easy computer algebra problem to check whether this newly computed table is permutation isomorphic to the stored $\mathbb{A} \mathbb{R L} \mathbb{A}$ table for a group with this name, including the stored power map.

We were able to apply this strategy to all but four simple groups $S$ contained in the $\mathbb{A} \mathbb{T L} \mathbb{A}$ and found no discrepancy with the stored tables. We summarise our main result.

THEOREM 3.1. Let $G$ be a bicyclic extension of a simple group whose character table is given in the $\mathbb{A} \mathbb{T L} \mathbb{A}$, and different from $J_{4}, 2 .{ }^{2} E_{6}(2), 2 .{ }^{2} E_{6}(2) .2, B, 2 . B$ and $M$. The character table of $G$ has been automatically recomputed and found to agree with the one stored in the character table library of GAP.

(See the remarks in the introduction about the relationship between the GAP tables and the printed $\mathbb{A} T L \mathbb{A}$ S tables.)

Details of the computations underpinning this theorem can be found at [5], including the group generators that were used. The character tables were computed using Magma 2.21-4 on a 2.9 GHz machine with 1TB Ram. Most of the constructions were routine and used few resources, both in time and memory; those with composition factors ${ }^{2} E_{6}(2), F_{3}$ and $T h$ were challenging, the last taking 988923 seconds.

3.5. $J_{4}, 2 .{ }^{2} E_{6}(2)$, and $2 .{ }^{2} E_{6}(2) .2$. We were able to compute the character tables of $J_{4}, 2 .{ }^{2} E_{6}(2)$, and $2 .^{2} E_{6}(2) .2$ using a combination of standard character theoretic methods (induction from subgroups, LLL reduction, and the enumeration of orthogonal embeddings); published information about conjugacy classes and subgroups; and character tables verified in Theorem 3.1 for particular subgroups. These calculations are described in full in [4. Specifically, the following information suffices for the computations.

$J_{4}$ : The description of conjugacy classes of $J_{4}$ as given in 10; the character table of the subgroup of type $2^{11}: M_{24}$; this subgroup is the unique primitive permutation group on $2^{11}$ points of that order that can be embedded into $J_{4}$.

$2 .{ }^{2} E_{6}(2)$ : The outer automorphism group of ${ }^{2} E_{6}(2)$, a symmetric group on three points, acts faithfully on the classes of ${ }^{2} E_{6}(2)$; and $2 .{ }^{2} E_{6}(2)$ contains subgroups of type $2 . F_{4}(2)$; and ${ }^{2} E_{6}(2)$ contains subgroups of types $F_{4}(2)$, $F i_{22}, 3 \times \mathrm{U}_{6}(2)$, and $\mathrm{O}_{10}^{-}(2)$; the character tables of the preimages of these subgroups under the natural epimorphism from $2 .^{2} E_{6}(2)$.

$2 .^{2} E_{6}(2) .2$ : The character tables of subgroups of types $2 \times F_{4}(2) \times 2$ and $3 \times 2 . \mathrm{U}_{6}(2) .2$. The three character tables agree with the corresponding tables in GAP's character table library.

3.6. $B, 2 . B$ and $M$. The tables of the remaining three groups excluded in the theorem are out of reach to our methods; their verification will be considered by others.

Remark 3.2. Note that the Frobenius-Schur indicators of characters are not stored in the GAP tables, but recomputed when needed. But the indicators contained in the old "Cambridge format" files were checked at the time of their conversion into GAP and so no undocumented errors should exist. 
REMARK 3.3. The $\mathbb{A} \mathbb{T L} \mathbb{A}$ contains all bicyclic extensions of simple groups of Lie type possessing an exceptional Schur multiplier, with the sole exception of some extensions of ${ }^{2} E_{6}(2)$. More precisely, none of the bicyclic extensions with one of the extensions being of degree 3 are given. For many applications, it is useful to know those character tables as well.

To our knowledge, the current status for $S={ }^{2} E_{6}(2)$ is as follows. Frank Lübeck has computed the character table of $3 . S$ using character theoretic methods: Deligne-Lusztig theory contributes some information about faithful characters, and this suffices for completing the whole character table. The table of $6 . S$ can be computed from the tables of $2 . S$ and $3 . S$ automatically; the usual heuristics - form tensor products and apply LLL reduction - is surprisingly successful. Computing the tables of $3 . S .2$ and $6 . S .2$ is even easier since the outer automorphism acts nontrivially on the centre. The tables of $2 . S, 6 . S, 3 . S .2$, and $6 . S .2$ are available in $[3$.

\section{References}

[1] W. Bosma, J. Cannon, C. Playoust, The Magma algebra system. I. The user language. J. Symbolic Comput. 24 (1997), 235-265.

[2] J. Bray, D. Holt, C. Roney-Dougal, The Maximal Subgroups of the Low-Dimensional Finite Classical Groups. London Mathematical Society Lecture Note Series, 407. Cambridge University Press, Cambridge, 2013.

[3] T. Breuer, The GAP Character Table Library, Version 1.2.2; 2013, available at http://www.math.rwth-aachen.de/ Thomas.Breuer/ctbllib.

[4] T. Breuer, Constructing the ordinary character tables of some Atlas groups using character theoretic methods. In preparation.

[5] T. Breuer, Input data for the verification of Atlas character tables, available at http://www.math.rwth-aachen.de/ Thomas.Breuer/atlasverify.

[6] T. Breuer, S. P. Norton, Improvements to the Atlas. Appendix 2 (pp. 297-327) in 11.

[7] J. H. Conway, R. T. Curtis, S. P. Norton, R. A. Parker, R. A. Wilson, Atlas of Finite Groups. Clarendon Press, Oxford, 1985.

[8] G. Frobenius, Über die Charaktere der mehrfach transitiven Gruppen. Berl. Ber. (1904), 558-571 (see Ges. Abh. III, 335-348).

[9] D. Gorenstein, R. Lyons, R. Solomon, The Classification of the Finite Simple Groups. Number 3. Mathematical Surveys and Monographs, American Mathematical Society, Providence, RI, 1998.

[10] Z. JANKO, A new finite simple group of order $86 \cdot 775 \cdot 571 \cdot 046 \cdot 077 \cdot 562 \cdot 880$ which possesses $M_{24}$ and the full covering group of $M_{22}$ as subgroups. J. Algebra 42 (1976), 564-596.

[11] C. Jansen, K. Lux, R. Parker, R. Wilson, An Atlas of Brauer Characters. London Mathematical Society Monographs. New Series, vol. 11, The Clarendon Press Oxford University Press, New York, 1995.

[12] P. Kleidman, The maximal subgroups of the Chevalley groups $G_{2}(q)$ with $q$ odd, the Ree groups ${ }^{2} G_{2}(q)$, and their automorphism groups. J. Algebra 117 (1988), 30-71.

[13] P. Kleidman, The maximal subgroups of the Steinberg triality groups ${ }^{3} D_{4}(q)$ and of their automorphism groups. J. Algebra 115 (1988), 182-199.

[14] G. A. Miller, Sur l'énumération des groupes primitifs dont le degré est inférieur à 17. C. R. Acad. Sci. Paris Sér. I Math. 124 (1897), 1505-1508.

[15] S. P. NoRTON, Improvements to the Atlas-II, http://brauer.maths.qmul.ac.uk/ Atlas/info/fullatlasmods.html.

[16] S. P. Norton, R. A. Wilson, The maximal subgroups of $F_{4}(2)$ and its automorphism group. Comm. Algebra 17 (1989), 2809-2824.

[17] G. Schneider, Dixon's character table algorithm revisited. Computational group theory, Part 1. J. Symbolic Comput. 9 (1990), 601-606.

[18] I. Schur, Untersuchungen über die Darstellung der endlichen Gruppen durch gebrochene lineare Substitutionen. J. Reine Angew. Math. 132 (1907), 85-137. 
[19] I. Schur, Über die Darstellung der symmetrischen und der alternierenden Gruppe durch gebrochene lineare Substitutionen. J. Reine Angew. Math. 139 (1911), 155-250.

[20] W. A. Simpson, J. S. Frame, The character tables for $\operatorname{SL}(3, q), \operatorname{SU}\left(3, q^{2}\right), \operatorname{PSL}(3, q)$, $\operatorname{PSU}\left(3, q^{2}\right)$. Canad. J. Math. 25 (1973), 486-494.

[21] C. C. Sims, Computational methods in the study of permutation groups. Computational Problems in Abstract Algebra, (Proc. Conf., Oxford, 1967), pp. 169-183, Pergamon, Oxford, 1970.

[22] M. Suzuki, On a class of doubly transitive groups. Ann. of Math. (2) 75 (1962), 105-145.

[23] The GAP Group, GAP - Groups, Algorithms, and Programming, Version 4.7.7; 2015, http://www.gap-system.org.

[24] W. R. Unger, Computing the character table of a finite group. J. Symbolic Comput. 41 (2006), 847-862.

[25] R. A. Wilson, The geometry and maximal subgroups of the simple groups of A. Rudvalis and J. Tits. Proc. London Math. Soc. 48 (1984), 533-563.

[26] R. A. Wilson, Maximal subgroups of sporadic groups. These Proceedings.

Lehrstuhl D für Mathematik, RWTh Aachen, 52062 Aachen, Germany.

E-mail address: thomas.breuer@math.rwth-aachen.de

FB Mathematik, TU Kaiserslautern, Postfach 3049, 67653 Kaiserslautern, GerMANY.

E-mail address: malle@mathematik.uni-kl.de

Department of Mathematics, University of Auckland, Private Bag 92019, AuckLAND, New Zealand

E-mail address: e.obrien@auckland.ac.nz 